\title{
The effect of the dye neutral red on a strain of Staphylococcus aureus in the presence of sunlight
}

\author{
B. CHATTOPADHYAY 1
}

From the Department of Bacteriology, St Bartholomew's Hospital, London

SYNOPSIS A strain of Staphylococcus aureus was isolated, the growth of which in the presence of neutral red was inhibited by sunlight. This phenomenon was not shown by any of a number of other strains of the same or different genera.

The present study was undertaken after the accidental finding of a peculiar effect of sunlight on a particular strain of Staphylococcus aureus on MacConkey agar plate.

In May 1973, a technician after inoculating an ear swab on a blood agar (Oxoid) plate and a MacConkey agar (Oxoid) plate, left both on the bench in the presence of sunlight for about 20 minutes before incubation. When the plates were examined the following morning, an unusual appearance was noted on the MacConkey agar plate only. The patient's name and the date of inoculation has been written in black marking ink on the underside of the petri dish; the plates had been left inverted on the bench and this writing had cast a shadow on the culture medium and inoculum beneath. Where the shadow had been cast a sharply delineated, luxuriant growth of Staphylococcus aureus occurred on incubation, but elsewhere on the plate the growth was scanty (figure).

\section{Materials and Methods}

Apart from the property described here, the strain (laboratory number 3715/73) was a typical coagulase-positive Staphylococcus aureus of phage type NT/29/71. It was sensitive to erythromycin, tetracycline, cloxacillin, and neomycin, but resistant to penicillin by the disc diffusion method. Also tested for inhibition by sunlight in the presence of neutral red were other 28 strains of Staphylococcus aureus, nine strains of Staphylococcus albus, four strains of Proteus, and four strains of Pseudomonas species isolated in the diagnostic laboratory.

The ability of the strains to grow on the media

'Present address: Department of Microbiology, Whipps Cross Hospital London E11.

Received for publication 22 February 1974.

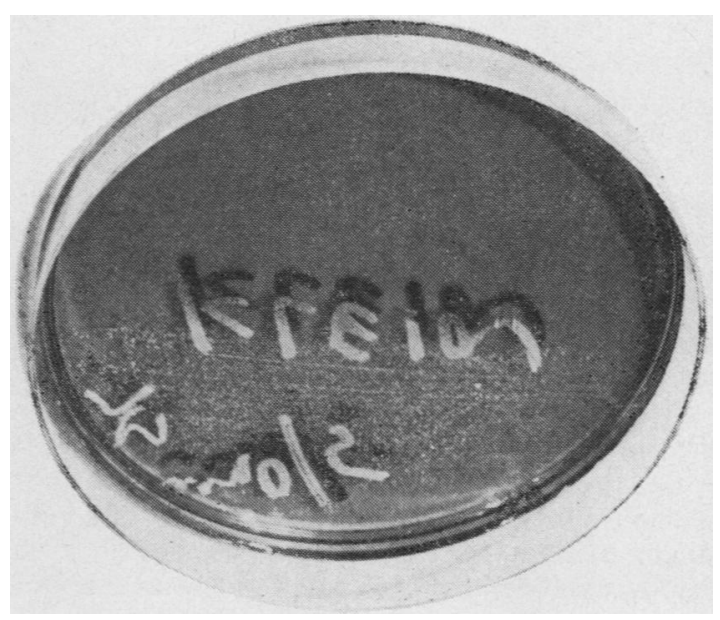

Fig The appearance of the original MacConkey agar plate inoculated with the particular strain of Staphylococcus aureus under test. This plate was exposed to sunlight for 20 minutes and incubated overnight at $37^{\circ} \mathrm{C}$.

listed in table I was tested by pouring the media into presterilized petri dishes $90 \times 15 \mathrm{~mm}$ in diameter (Sterilin Limited), and flooding them with different strains under test, the size of the inoculum being $10^{5}$ organisms $/ \mathrm{ml}$. The plastic plates were labelled with a Pentel felt tip pen containing blue, black, or red ink. The plates were inverted and exposed to sunlight for 10 to 60 minutes as in the original observation so that felt-pen labels cast a shadow on the medium beneath. They were then incubated at $37^{\circ} \mathrm{C}$ overnight.

In an attempt to reproduce the phenomenon artificially, Spekker filters were placed over the plates during exposure to sunlight or ultraviolet 
Medium Composition of Medium

No.

Nutrient agar (Oxoid, code CM 3, Lab-Lemco powder, yeast extract, peptone, sodium chloride, agar no. 3)

Blood agar base (Oxoid, code CM 55, Lab-Lemco, peptone, sodium chloride, agar no. 3)

Salt agar (blood agar base as above $+5 \%$ sodium chloride)

Cystine, lactose electrolyte-deficient medium (CLED-Oxoid, code CM 301, peptone, Lab-Lemco, tryptone, lactose, L-cystine,

bromothymol blue, agar no. 1)

Blood agar (Oxoid, code PM 1055B-ready poured)

Lysed blood agar (Oxoid-DST agar, code PM 1261LB, ready poured)

Chocolate agar (blood agar base + sterile heated defibrinated horse blood)

Blood agar base $+0.5 \%$ bile salt

Blood agar base $+1 \%$ lactose

Blood agar base $+0.5 \%$ bile salt $\div 1 \%$ lactose

Blood agar base $+0.007 \%$ neutral red

Blood agar base $+0.5 \%$ bile salt $+0.007 \%$ neutral red

Blood agar base $+1 \%$ lactose $+0.007 \%$ neutral red

MacConkey agar (Oxoid CM 76, peptone, lactose, bile salt, neutral red, agar no. 3)

Table I Composition of media tested for inhibition of growth by sunlight

light $(253 \cdot 7 \mathrm{~nm}$, energy emitted 37 microwatt $/ \mathrm{sq} \mathrm{cm})$. Control plates were set up without having Spekker filters placed over them in both experiments.

\section{Results}

The figure shows the appearance of a MacConkey agar plate inoculated with the original strain of Staphylococcus aureus exposed to sunlight for 20 minutes and incubated overnight at $37^{\circ} \mathrm{C}$. There was dense growth on areas shadowed by the felt-pen writing on the plate.

This effect was not found when this organism was inoculated on media 1-10 (table I). Table II shows that when the components of MacConkey medium were separated, only combinations containing neutral red showed the phenomenon. A shadow cast over the sunlit MacConkey medium for up to an hour before inoculation with this particular staphylococcus failed to reproduce the phenomenon.

Plates shaded from sunlight by filters also showed the phenomenon which was not affected by the colour of the filters or of the ink marker.

When the plates were exposed directly to ultraviolet light with the lids off the usual bactericidal effect of ultraviolet light was observed. The plates were sterile on incubation after exposure for

\begin{tabular}{ll}
\hline Medium & $\begin{array}{l}\text { Phenomenon } \\
\text { Demonstrated }\end{array}$ \\
\hline BAB $^{1}+0.5 \%$ bile salt & No \\
BAB $+1 \%$ lactose & No \\
BAB $+0.007 \%$ neutral red & Yes \\
BAB $+0.5 \%$ bile salt $+1 \%$ lactose & No \\
BAB $+0.5 \%$ bile salt $+0.007 \%$ neutral red & Yes \\
BAB $+1 \%$ lactose $+0.007 \%$ neutral red & Yes \\
\hline
\end{tabular}

Table II Effect of constituents of MacConkey agar to demonstrate the phenomenon with Staphylococcus aureus

${ }^{1}$ BAB = blood agar base (Oxoid, as described above)
30 to 40 minutes. When plates were exposed to ultraviolet irradiation exactly in the same way as described above for sunlight no effect could be shown.

Table III shows the influence of time on the appearance of the phenomenon.

\begin{tabular}{lll}
\hline $\begin{array}{l}\text { Time of Exposure of } \\
\text { Plate to Sunlight } \\
\text { (minutes) }\end{array}$ & $\begin{array}{l}\text { Growth on Exposed Area } \\
\text { of MacConkey Plate }\end{array}$ & $\begin{array}{l}\text { Phenomenon } \\
\text { Demonstrated }\end{array}$ \\
\hline $10-20$ & Good & No \\
$20-30$ & Moderate & Yes \\
$30-40$ & Scanty & Yes \\
$40-50$ & Very scanty & Yes \\
$50-60$ & None & Yes
\end{tabular}

Table III Effect of time of exposure to sunlight on occurrence of growth of Staphylococcus aureus

\section{Discussion}

An inhibitory effect of the dye neutral red in the presence of sunlight on a particular strain of Staphylococcus aureus was observed. It has been reported in the past that neutral red may exert an inhibitory effect on the growth of Escherichia coli $\mathrm{N}$ in samples of water and that the action is most $N$ probably on the bacterial cell wall (Childs and $\mathrm{\omega}^{-}$ Allen, 1953). Also it has been shown that the preexposure of nutrient agar plates to sunlight inhibits the growth of a number of strains of Staphylococcus aureus (Waterworth, 1969). Had the effects been $\stackrel{\Phi}{q}$ due to the protection of bacteria from sunlight by 0 the writing on the plastic petri dish or superimposed filters, the same effect would have been produced on any transparent medium capable of supporting the growth of this or any other strains of staphylococci. But the effect was seen only on media containing neutral red and astonishingly so far only this 
particular strain has shown this phenomenon. I am most grateful to Professor F. W. O'Grady for Evidently dye and sunlight worked together in- his suggestions and guidance and to Dr E. J. Shaw, hibiting this unusual strain.

Plenty of sunshine in the months of June and to some extent in August enabled us to carry out these tests. Unfortunately, the effects of different dyes or of glass petri dishes could not be tested due to cloudy weather. Also the actual difference in the intensity of sunlight and ultraviolet light used in these tests is not known. Further attempts to reproduce the phenomenon by exposure to artificial light sources of different intensities are in progress. senior lecturer, for her help in preparation of this manuscript. I would like to thank Mr R. Andrew, the chief technician, for drawing my attention to this peculiar phenomenon. I acknowledge with thanks the secretarial help of Mrs L. U. Manning.

\section{References}

Childs, E., and Allen, L. A. (1953). Improved methods for determining the most probable number of Bacterium coli and of Streptococcus faecalis. J. Hyg. (Lond.), 51, 468-477.

Waterworth, P. M. (1969). The action of light on culture media. J. clin. Path., 22, 273-277. 